\title{
Quantitative mass spectrometry in proteomics
}

\author{
Marcus Bantscheff • Bernhard Kuster
}

Published online: 24 July 2012

(C) Springer-Verlag 2012

Investigating living systems at the protein level is continuing to provide important insights into many biological processes across all kingdoms of life. Mass spectrometry based proteomics has fundamentally changed the way in which biological systems are interrogated because of its ability to measure thousands of proteins and post-translational modifications in parallel. This enables investigations at all levels of biological complexity ranging from protein complexes to human patient populations. While five years ago, the majority of proteomic experiments mostly enumerated the protein constituents of a biological system, quantitative measurements are at the heart of practically every proteomic study today. This shift was once more significantly driven by developments in mass spectrometry which is now the de-facto standard for quantitative measurements in proteomics. Alongside, the miniaturization of associated sample preparation and separation techniques as well as the adoption and further development of data analysis strategies from other-omics research have helped transform proteomics from a qualitative to a quantitative science.

At the same time, the areas of life science research to which proteomic methods are being applied are expanding rapidly. While a few years ago, most of the successful proteomic work was performed in rather simple models such as unicellular organisms or cell lines derived of animal and plant origin, it is becoming increasingly possible to work with primary samples including that of human patients. However, scientists engaged in such studies had to realize that the enormous molecular

Published in the topical issue Quantitative Mass Spectrometry in Proteomics with guest editors Bernhard Kuster and Marcus Bantscheff.

M. Bantscheff

Analytical Sciences, Cellzome AG,

Meyerhofstrasse 1,

69117 Heidelberg, Germany

B. Kuster $(\square)$

Chair of Proteomics and Bioanalytics,

Technische Universitaet Muenchen,

Emil Erlenmeyer Forum 5,

85354 Freising, Germany

e-mail: kuster@tum.de complexity and the dynamic nature of proteomes posed much larger challenges than those encountered for either genome or transcriptome studies. In particular, issues related to splice variants, post-translational modifications, dynamic range of protein expression spanning 10 orders of magnitude, protein stability, transient protein associations, and dependence on cell type or physiological state etc. are still limiting our technical ability to characterize proteomes comprehensively and reproducibly in a reasonable time. Unsurprisingly, numerous experimental strategies and schemes have been devised to address the many challenges and, as in any developing field, some have come and gone, while others have been adopted more broadly. It is probably safe to assume that we have not yet seen the end of the technical developments which, in turn, will allow more and more difficult biological systems to be approached by proteomics.

Despite all the hurdles, mass spectrometry based quantitative proteomics increasingly impacts life science research in many areas including protein expression profiling, the analysis of signaling pathways or the development of protein biomarker assay systems to name a few. It is important to note that in each area, distinct scientific questions are being asked and, therefore, distinct proteomic approaches will have to be applied, many of which vary widely in their versatility, technical maturity, difficulty, and expense. Consequently, we must recognize that some scientific questions are, and will remain, much harder to answer by proteomics than others. Still, the strong trend towards the alternative scientific paradigm of system-level interrogation of biological phenomena is unstoppable and will without doubt continue to provide important complementary views to traditional and still highly successful hypothesis-driven research.

In this special issue on quantitative mass spectrometry in proteomics, we have assembled a group of reputed scientists who all have significant statements to make regarding the topic of this issue. There are eight reviews that provide an in-depth appreciation of a state-of-the-art technology alongside six original research articles highlighting some of the current trends. We can of course not claim to cover the field comprehensively, but do believe that the content of this special issue has a lot to 
offer, both for newcomers as well as experts in the field of mass spectrometry based proteomics.

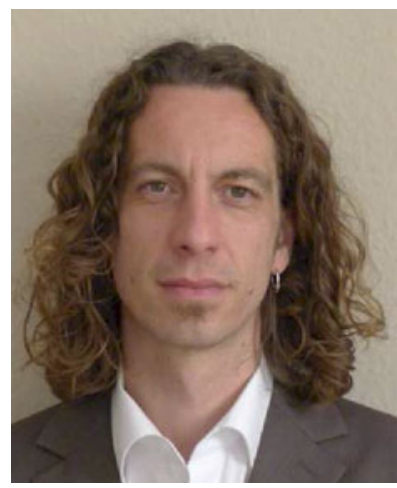

\section{Marcus Bantscheff}

graduated in Chemistry from the University of Konstanz and obtained his $\mathrm{PhD}$ degree from the University of Rostock, working with Prof. Glocker on structure-function correlation of bacterial response regulator proteins utilizing mass spectrometric and protein chemistry methods. As a postdoctoral fellow at the Proteome Center in Rostock, Marcus was involved in setting up a proteomics unit and focused on the analysis of synovial fluids and tissue samples derived from rheumathoid arthritis patients and CIA mice. At Cellzome since 2002, Dr. Bantscheff's research interests have focused on quantitative proteomics and chemoproteomic technologies. Marcus is an inventor on several patent applications including Cellzome's Kinobeads ${ }^{\mathrm{TM}}$ and Episphere ${ }^{\mathrm{TM}}$ technologies, author of more than 30 publications in scientific journals including Nature Biotechnology, Nature, and Nature Chemical Biology. He is the editor of a chemoproteomics methods compendium and reviewer for a number of scientific journals.

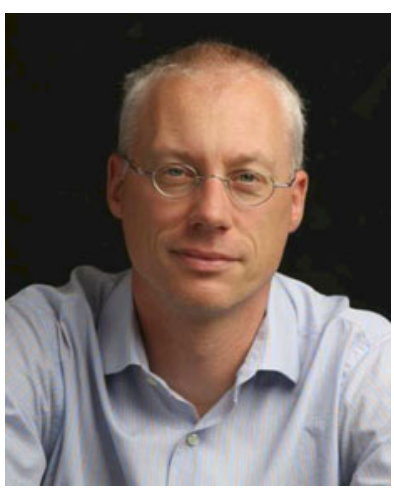

Bernhard Kuster

is a chemist by training and obtained an MSc in 1994 from the University of Cologne. He received his $\mathrm{PhD}$ in Biochemistry from the University of Oxford in 1997 and spent his PostDoc years with Matthias Mann at the European Molecular Biology Laboratory in Heidelberg and at the University of Southern Denmark in Odense, Denmark. Between 2000 and 2007, Professor Kuster held several positions at the biotech firm Cellzome, most recently that of VP Analytical Sciences and Informatics. In 2007, he was appointed Full Professor and Chair of Proteomics and Bioanalytics at the Technische Universität München, TUM where he is currently also the Head of the Department for Biosciences, an executive of the TUM graduate school Experimental Biomedicine and a member of the Excellence Cluster CIPSM. Professor Kuster is an inventor of several patent applications including Cellzome's Kinobeads ${ }^{\mathrm{TM}}$ and has published more than 70 scientific articles in highly reputed journals. He also serves on the editorial and scientific advisory boards of several journals and university institutes. His current research interests include functional proteomics, chemical biology and cancer drug and biomarker discovery. 